University of Nebraska - Lincoln DigitalCommons@University of Nebraska - Lincoln

1995

\title{
Host Age as a Determinant of Naturally Acquired Immunity to Plasmodium falciparum
}

J. Kevin Baird

ALERTAsia Foundation, jkevinbaird@yahoo.com

Follow this and additional works at: http://digitalcommons.unl.edu/publichealthresources

Baird, J. Kevin, "Host Age as a Determinant of Naturally Acquired Immunity to Plasmodium falciparum" (1995). Public Health Resources. 417.

http://digitalcommons.unl.edu/publichealthresources/417

This Article is brought to you for free and open access by the Public Health Resources at DigitalCommons@University of Nebraska - Lincoln. It has been accepted for inclusion in Public Health Resources by an authorized administrator of DigitalCommons@University of Nebraska - Lincoln. 


\title{
Host Age as a Determinant of Naturally Acquired Immunity to Plasmodium falciparum
}

\author{
J.K. Baird
}

The usual course of infection by Plasmodium falciparum among adults who lack a history of exposure to endemic malaria is fulminant. The infection in adults living with hyper- to holoendemic malaria is chronic and benign'. Naturally acquired immunity to falciparum malaria is the basis of this difference. Confusion surrounds an essential question regarding this process: What is its rate of onset? Opinions vary because of disagreement over the relationships between exposure to infection, antigenic polymorphism and naturally acquired immunity. In this review, Kevin Baird discusses these relationships against a backdrop of host age as a determinant of naturally acquired immunity to falciparum malaria.

Naturally acquired immunity, as used here, refers specifically to a diminished frequency and density of parasitemia by Plasmodium falciparum in adults relative to children where hyper- to holoendemic malaria prevails. It may be characterized as an 'antiparasite' immune process. However, progressively fewer and less-severe attacks of malarial fevers and chills attend diminishing susceptibility to parasitemia. There may be as yet poorly understood 'antidisease' immune processes that operate separately from the conspicuous antiparasite function of naturally acquired immunity. However, immune restraint of blood-stage parasites per se certainly confers protection from disease. In this sense, naturally acquired immunity includes both antiparasite and antidisease processes.

Many investigators consider 'antidisease' immunity synonymous with the process called 'antitoxic' immunity by MacGregor ${ }^{2}$. Antitoxic immunity appears in young children (2-3 years old) exposed to heavy

J. Kevin Baird is at the Department of Parasitology US Naval Medical Research Unit \#2, Jakarta, Box 3, American Embassy APO AP 96520-8132, USA. Tel: +62 $21 \mathbf{4 2 0}$ 7854, Fax: +62 21424 4507, e-mail: NAMRU2@WRAIR-EMHI.ARMY.MIL infection pressure since birth ${ }^{2}$. Asexual parasitemias of extraordinary density without apparent illness marks antitoxic immunity. This immunity somehow stays the sequelae of blood-stage infection without apparent effect upon the parasite itself. In contrast, the naturally acquired immunity of adults in endemic areas precludes high-density parasitemias and attendant disease. This article excludes discussion of antitoxic immunity. It examines the basis of the differences in susceptibility to more frequent and higher density asexual parasitemias of $P$. falciparum between older children (5-15 years old) and adults.

Empirical observation has revealed much of what is understood of naturally acquired immunity. Exposure to relatively heavy infection pressure is required; naturally acquired immunity fails to develop where malaria is epidcmic, hypoendemic or mesoendemic. Naturally acquired immunity fails to develop or, once developed, is lost with interrupted exposure (eg. with seasonal malaria or extended travel out of an endemic area). Longitudinal studies where malaria is holoendemic have consistently shown evidence of asexual parasitemias in virtually all adults; naturally acquired immunity does not sterilize. Children in hyper- to holoendemic areas consistently exhibit higher-grade and more frequent asexual parasitemias with clinical attacks compared to adults in the same area. The differences between children and adults are not abrupt but progress gradually. MacGregor ${ }^{3}$ has reviewed naturally acquired immunity to plasmodium parasites.

The development of naturally acquired immunity has been considered a slow process, ie. requiring the years of life between infancy and adulthood ${ }^{4-7}$. Studies published since the 1930s until now express this without equivocation. It is an accepted convention. Although the process evidently occurs slowly in people born and raised in a malarious area, no quantitative body of evidence proves the requirement for many years of 


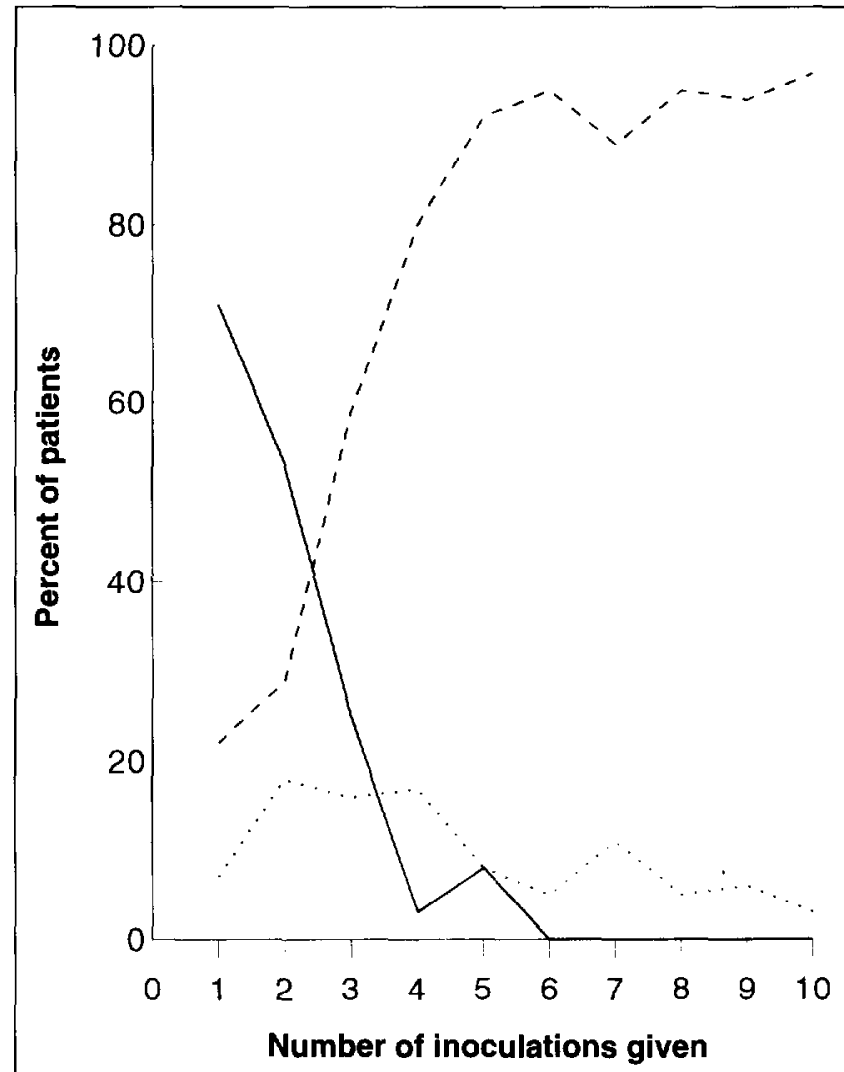

Fig. I. The results of a total of 1066 separate blood-stage inoculations between 1926 to 1932 at the Socola Malariatherapy Centre in Jassy, Romania 15 are presented. These data show the onset of homologous immunity among 95 (two inoculations) to 36 (ten inoculations) patients treated with the same strain of $P$. falciparum ${ }^{15}$; 544 patients received just one inoculation. No fever, no parasitemia (dashed curve); parasitemia only (dotted curve); fever and parasitemia (solid curve).

uninterrupted heavy exposure to antigen. Indeed, there is dissent on the issue. There are those who assert that naturally acquired immunity may develop after fewer than ten episodes of infection within 12 to 24 months $^{8-11}$. Although these two opinions seem to be diametrically opposed, a simple principle may reconcile them; a few episodes of malaria are sufficient to develop naturally acquired immunity in adults, whereas the immune systems of children may be constitutionally less capable of mounting a protective response against the parasite. If this is true, the onset of naturally acquired immunity may be governed primarily by recent exposure to infection and intrinsic immune factors related to the age of the host $t^{8,10}$.

\section{The basis of conventional naturally acquired immunity}

With the exception of a few elegant demonstrations of passive transfer of naturally acquired immunity reviewed clsewhere ${ }^{12}$, the certainty that it exists at all vanishes without the epidemiological data based on microscopic examination of stained blood films gathered from people living in endemic areas. The cellular and molecular bases are not sufficiently well understood to yield an assay that measures the degree of naturally acquired immunity in any given individual or population. Survey by bloodfilm examination and spleen palpation remain the sole means of identifying and measuring naturally acquired immunity.
Robert Koch pioneered the use of stained bloodfilms in studying the epidemiology of malaria. In 1900, he noticed diminishing frequency and density of parasitemia with increasing age among people in the hyperendemic Ambarawa Valley of Central Java ${ }^{13}$. Koch also surveyed Sukabumi in West Java, where there was considerably less malaria, and the frequency of parasitemia was not age related. The contrasting patterns of frequency of parasitemia among age groups at these two sites suggested to Koch that the cumulative effect of lifelong exposure to heavy infection pressure endowed protective immunity. The age-related pattern of parasitemia in heavily endemic areas has been a consistent observation over the years ${ }^{14}$, and it remains the basis of the convention that naturally acquired immunity develops slowly over many years with heavy exposure to infection.

Malariotherapy of syphilitic patients in the 1920 s set the stage for rational analyses of acquired immunity. These studies established the relatively rapid onset of immunity to homologous strains of parasites (4-10 exposures $)^{15-17}$. The rate of onset of homologous immunity was rapid over the first five exposures and thereafter slowly improved to nearly complete immunity after ten exposures ${ }^{15}$ (Fig. 1). Although Ciuca et al. were careful to point out that their study subjects lived where malaria was endemic (Romania), their results do not differ substantially from studies using patients with no history of exposure.

Malariologists conducting studies in syphilitic patients recognized the difficulty in reconciling the rapid rate of development of immunity in the clinic with the apparently very slow rate in endemic areas. Studies with heterologous strains of parasite provided a plausible answer; patients with homologous immunity remained at least partially susceptible to heterologous strains of parasite. Heterologous susceptibility in the clinic suggested that the antigenic diversity of wild parasites explained the relatively slow development of natural immunity acquired in the field.

Brown and Brown ${ }^{18}$ demonstrated the existence of variant antigenic types (VATs) on the surface of red blood cells infected by Plasmodium knowlesi in rhesus monkeys. Similar observations were made in $P$. fragile in toque monkeys ${ }^{19}$, and in $P$. falciparum in squirrel monkeys ${ }^{20}$. Extensive clonal antigenic variation by $P$. falciparum has been established ${ }^{21,22}$. Studies with monoclonal antibodies or immune sera have demonstrated extreme allelic diversity in blood stages of wild malaria parasites ${ }^{23} 26$. Tandem repeats of oligopeptides (which often compose immunodominant epitopes) apparently promote multiple crossreactivities, and this may be an evolutionary advantage that confounds host immune defenses 27,28 . All this provided a plausible molecular explanation for the slow development of naturally acquired immunity, but evidence linking any particular antigenic polymorphism to human susceptibility to natural infection has been elusive.

\section{Limitations of conventional naturally acquired immunity}

Reports of allelic or clonal antigenic polymorphism in plasmodium parasites often present such findings in the context of explaining the slow onset of naturally acquired immunity $3-6,19,20,25$. According to the conventional view of the development of naturally acquired 


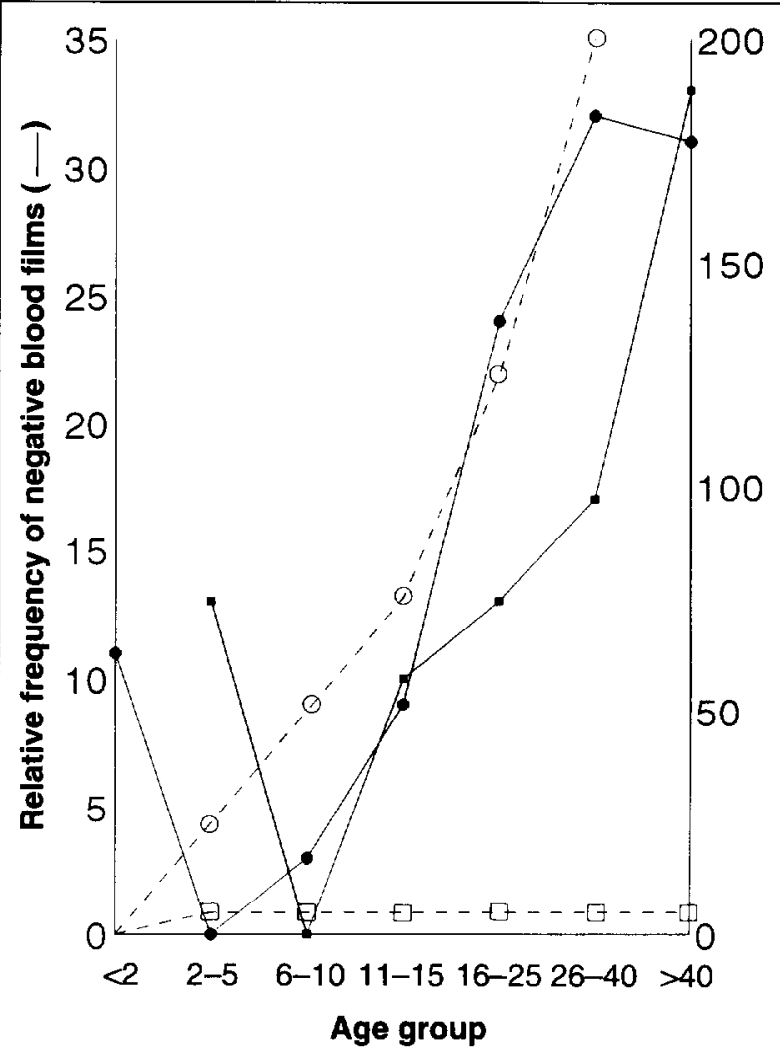

Fig. 2. The relationship between age, cumulative exposure (CE) (dashed curves) and an estimate of naturally acquired protective immunity (solid curves) expressed here by the statistic 'relative frequency of negative bloodfilms':

$$
\frac{\% \text { neg }_{a}-\% \text { neg }_{\left.\right|_{a}}}{\Sigma\left[\% \text { neg }_{a}-\% \text { neg }_{\text {a }}\right]} \times 100
$$

(where \%neg is the age-specific frequency of negative blood films, and \%neg $\mathrm{l}_{\mathrm{a}}$ is the lowest frequency of negative blood films among age groups). Cumulative exposure is the estimated number of infections per person, and was calculated assuming an attack rate of three infections per person year, which are known to occur in the Arso area of Irian Jaya ${ }^{59}$. The migrant population [closed squares (relative frequency of negative blood film) and open squares (CE)] composed residents of Arso $V$ in Irian Jaya, Indonesia sampled after 20 months of exposure to infection ${ }^{10}$. Data from the 'native' population [closed circles (relative frequency of negative blood films) and open circles (CE)] reported by McGregor and Smith ${ }^{35}$ from people with lifelong exposure in The Gambia.

immunity, antigenic polymorphism governs susceptibility to infection for many years of heavy exposure. The parasite presumably manages evasion of an efficacious immune response through sheer diversity of epitopes. The chronically exposed host supposedly accumulates a repertoire of memory and effector cells capable of controlling infection by any given strain or variant of parasite. This seems logical, but the hypothesis assumes that naturally acquired immunity only develops slowly over the years between infancy and adulthood. In a quantitative sense, about 100 infections and associated waves of recrudescences seem necessary (age 0-20 years, assuming five infections per year where malaria is hyper- or holoendemic). Is this true?

Epidemiological studies of human populations living in endemic areas constitute the basis of the assumption that naturally acquired immunity develops slowly.

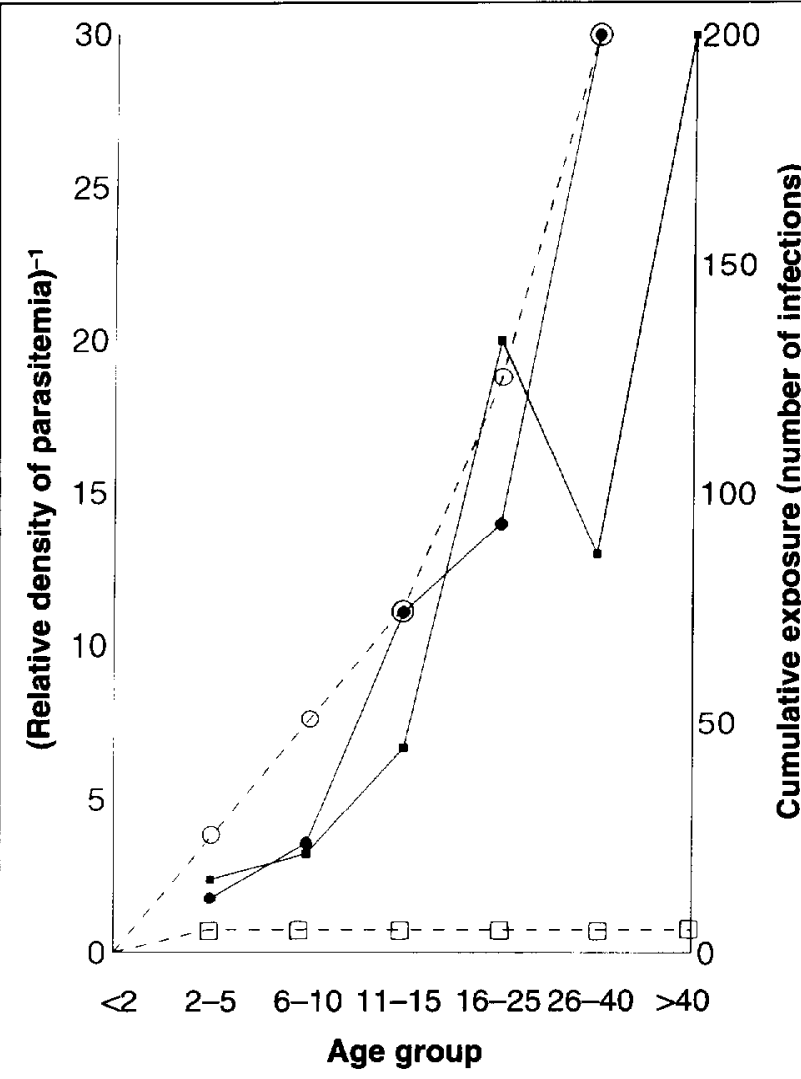

Fig. 3. The relationship between age, cumulative exposure (CE) (dashed curves) and an estimate of naturally acquired protective immunity (solid curves) expressed here by the inverse of the statistic 'relative density of parasitemia':

$$
\frac{C_{a}-C_{1 a}}{\Sigma\left[C_{a}-C_{l_{a}}\right]}
$$

(where $C_{a}$ is the mean asexual parasite count within age groups and $C_{l a}$ is the lowest mean asexual parasite count among the age groups). The cumulative exposures were calculated assuming an attack rate of three infections per person year, which are known to occur in the Arso area of Irian Jaya ${ }^{59}$. The migrant population (closed and open squares) comprised residents of Arso $V$ in Irian Jaya, Indonesia, sampled after 20 months of exposure to infection ${ }^{10}$. Data from the native population (closed and open circles) were taken from the report of McGregor and Smith ${ }^{35}$ describing malaria among lifelong residents of Keneba, The Gambia, in 1952.

Although nol often defined, 'slow' presumably refers to the consistent pattern of age-related prevalence and density of parasitemia, ie. gradually diminishing throughout life beyond 3-10 years of age. Many other parameters have been evaluated in the field: frequency and severity of symptoms of malaria, spleen enlargement, antibody titers and frequencies, and lymphocyte blastogenesis ${ }^{29-34}$. These studies also show increasing protection or 'immunity' (according to the parameter being measured) with increasing age. These patterns are consistent with a slow rate of onset of naturally acquired immunity in conjunction with cumulative exposure to infection over the course of life in an endemic area (Figs 2,3). However, these observations, per se, do not establish the cumulative effects of exposure as the basis of age-related changes in susceptibility to infection. Correlation between age and various measures of 


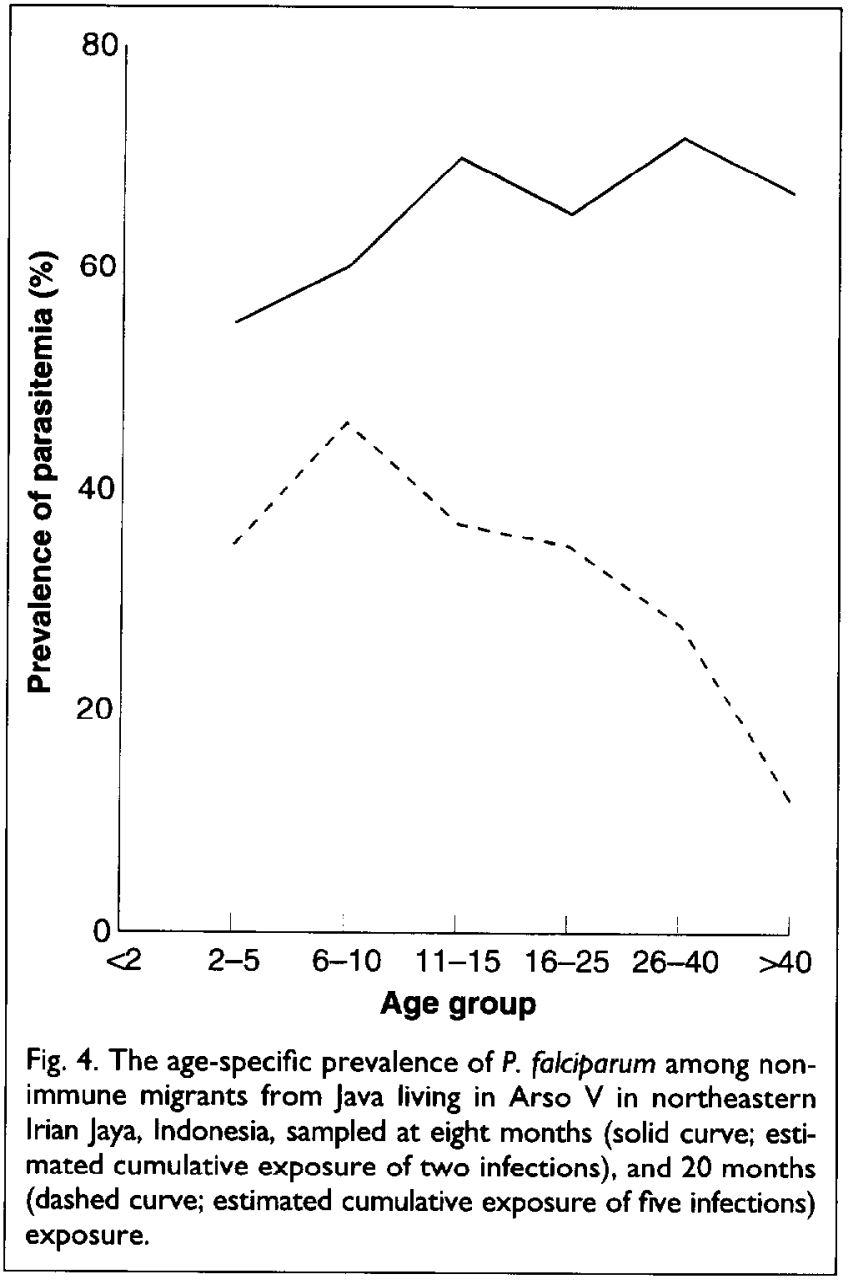

'immunity' does not prove that the onset of naturally acquired immunity is slow.

Conventional theory of naturally acquired immunity attributes the susceptibility of children and the relative immunity of adults to their differing cumulative experiences with the antigenic repertoire of wild parasites. In this context, the only essential difference between the immune systems of children and adults is their histories of exposure. The constitutional changes which occur with aging are assumed to exert no influence on the course of natural infection. However, among people living their entire lives in an endemic area, the effects of cumulative exposure cannot be separated from any possible effects of age itself. There has been no demonstration that this assumption, which underpins conventional hypotheses on naturally acquired immunity, is true.

One way to sort out the effects of cumulative exposure and age is to study naturally acquired immunity among people of all ages who are abruptly exposed to heavy infection pressure 8,10 (Figs 2,3). After just one year of residence in a hyperendemic area of Irian Jaya (Indonesian New Guinea) the frequency and density of parasitemia decreases with increasing age among newcomers from Java (Fig. 4). The patterns are parallel to those in people with lifelong residence in either Irian Jaya $^{8}$ or The Gambia (Figs 2,3) ${ }^{35}$. Age-related changes in humoral immune reactivity to ring-infected erythrocyte surface antigen of $P$. falciparum were also parallel between newcomers and lifelong residents ${ }^{8}$. Adult newcomers showed evidence of naturally acquired protection relatively quickly, whereas their children remained susceptible. Naturally acquired immunity in these adults developed independently of the cumulative effects of many years of heavy exposure (Figs 2,3 ).

\section{The hypothesis of age-dependent naturally acquired immunity}

If cumulative exposure to antigen does not account for the relative susceptibility of children and resistance of adults, then what does? Intrinsic immune factors that change with age may govern the degree of naturally acquired immunity following a brief period of heavy exposure to infection. The basis of the difference between children and adult Javanese transmigrants in Irian Jaya may be age-related immune factors unrelated to a history of chronic exposure to infection 8,10 .

The studies in Irian Jaya were conducted by a single laboratory using one ethnic group of non-immune people living in one region. The conclusions reached in these studies may not be generally applicable. Corroboration by other laboratories in other parts of the world is not yet available, although work in the early 1900 s in Sumatra ${ }^{36}$ and India ${ }^{37}$ showed similar patterns of agedependent, chronic exposure-independent, naturally acquired immunity. Schuffner ${ }^{36}$ and Christophers ${ }^{37}$ also wondered about the role of age itself in naturally acquired immunity, but both expressed doubts concerning the possibility of prior heavy exposure to infection by their study subjects.

The subjects from the studies in Irian Jaya came from Java, where the incidence of malaria is roughly one case per 10000 person years ${ }^{10}$ and has been this way since at least 1960 (Ref. 38). Thus, among 1000 transmigrants in a typical village in Irian Jaya (with a mean age of 20 years), probability favors only two infections in these 20000 person years on Java. Moreover, approximately $85 \%$ of infections occur among $0.5 \%$ of the population of Java living in fewer than 10 chronically endemic foci ${ }^{10}$. The risk of infection for $99.5 \%$ of people living on Java may actually be closer to two infections in 100000 person years (data from The Ministry of Health, Jakarta). This means just one prior exposure among 2500 transmigrants arriving in Irian Jaya (excluding those with a prior history of transmigration or residence in one of the known foci of endemic malaria on Java).

Extrinsic factors related to vector or host behavior may have explained the age-related pattern of parasitemia among the transmigrants in Indonesia. For example, vector feeding preference for children or agerelated differences in antimalarial drug consumption may have established the observed age-related patterns of prevalence and density of parasitemia. However, Fig. 4 illustrates an important observation: although children and adults were uniformly susceptible to infection early during exposure, protection later increased in an age-dependent manner. This pattern routinely emerges from populations of Javanese newcomers in Irian Jaya ${ }^{10}$. Naturally acquired immunity is apparently governed by recent exposure and the age of the host.

Evidence from several groups using outbred laboratory animals showed that age itself profoundly influences the course of infection by plasmodium parasites $^{39-43}$. Some of these studies demonstrated adoptive transfer of adult-like immunity to younger animals ${ }^{41,42}$. The age immunity against Plasmodium spp disappeared in some inbred strains of mice and rats, but not others. 
A cellular basis of age-dependent naturally acquired immunity is implicit in this work conducted 20 or more years ago. More-recent laboratory studies unrelated to infectious diseases have shown that many subtle changes in constitution of the immune system occur throughout life ${ }^{44-46}$. No particular change has yet been linked to susceptibility to infectious agents.

\section{Reconsidering laboratory evidence for conventional naturally acquired immunity}

If the hypothesis that age and recent heavy exposure govern efficacy of naturally acquired immunity is correct, then it must accord with the observations that form the basis of the conventional hypothesis for naturally acquired immunity. The original description of VATs in $P$. knowlesi was by Brown and Brown ${ }^{18}$. Reports describing clonal antigenic polymorphism in other species of Plasmodium often cite this work, emphasizing host susceptibility to successive VATs and the possible relationship to host susceptibility and the slow development of naturally acquired immunity. However, Brown and Brown ${ }^{18}$ noted another facet of their work; the existence of two levels of immunity, one VAT-specific and the other "transcending antigenic variation'. Strain-transcending immunity was also described against VATs of $P$. fragile by Handunnetti and colleagues ${ }^{19}$. Citing their own unpublished work and the reports of others, Brown and Brown ${ }^{18}$ noted that, '... children, unlike adults, are perhaps incapable of developing a generalized immunity effective against all variants'. They wondered whether childhood susceptibility to infection was the product of incomplete exposure to the available repertoire of immunogenic epitopes, or to a constitutional inability to mount a strain-transcending immune response. This question largely represents the hypothesis of an age-dependent naturally acquired immunity.

Investigators using in vitro agglutination of $P$. falciparum isolates from donors naturally exposed to infection have documented fine specificity in the humoral immune response to natural infection ${ }^{47-49}$. Sera from convalescent children usually agglutinate only the isolate taken at infection, whereas sera from 'immune' adults routinely agglutinate many isolates. The degree of crossreactivity among strains correlate with age of the serum donor ${ }^{48,50}$, and the capacity to agglutinate a single isolate increased with age ${ }^{30}$. Little is known of what is at work here. Strain-specific antibody may occur in adults, or antibody clones may exhibit broad crossreactivity. In other words, strain-transcending agglutination activity may be either the sum of relatively many strain-specific clonal antibodies or the product of relatively few clonal antibodies with broad specificities.

Newbold and colleagues ${ }^{51}$ reported predominantly strain-specific antibodies to parasite-derived neoantigens in pooled 'immune' sera. Among 18 pairs of 13 isolates, agglutinates of both isolates accounted for fewer than $20 \%$ of those observed microscopically. Those findings suggest that strain-transcending agglutination may be the sum of relatively many strain-specific antibodies. However, in the same experiment, 13 of 18 isolate pairs did exhibit at least some mixed agglutinates. This showed that crossreacting antibody was present in most instances ( $72 \%$ of pairs). The relatively low frequency of mixed versus homologous agglutinates reported by the authors may simply reflect a predictably lower affinity by crossreacting antibody. Higher affinity isolate-specific antibodies would be forecast to competitively inhibit binding and agglutination by lower affinity crossreacting antibodies. Which set of antibodies may play a predominant role in naturally acquired immunity remains uncertain.

If strain-specific antibody predominates in naturally acquired immunity, then the ability of immune sera to agglutinate many isolates must be attributed to cumulative exposure to, and long-term memory of, the antigenic repertoire of local parasites. On the other hand, some people with limited histories of exposure show surprisingly broad agglutination activity against geographically diverse isolates ${ }^{52}$. Does history of exposure correlate with the spectrum of strain recognition, agglutination or protective immunity? Studies of the immune response to infection by $P$. falciparum among adults with limited histories of exposure (see below) may help resolve this question.

\section{Reconsidering clinical evidence for conventional naturally acquired immunity}

If an age-dependent strain-transcending immunity exists, then non-immune adults (or those with homologous immunity) should relatively quickly show a protective response to heterologous infections. These effects occurred among syphilitic patients treated with $P$. falciparum. Patients with homologous immunity were usually partially susceptible to a heterologous strain ${ }^{53}$ (Fig. 5). Although lingering susceptibility has been viewed as supporting the importance of antigenic polymorphism in naturally acquired immunity, the decrease in susceptibility was no more or less marked than during early exposures to homologous strains (Fig. 1). In many instances, the degree of immunity to heterologous strains rivalled that against the homologous strain $^{54}$ (Fig. 6). In one series, three patients were initially infected by a Colombian strain of $P$. falciparum and then reinfected by a Thai strain. The second infection prompted an immediate rise in antibodies to the Colombian isolate ${ }^{55}$. The parasitemia cleared spontaneously after antibodies to the Thai isolate finally appeared. Adult patients with a history of exposure to just a single strain of parasite often demonstrated a high capacity to suppress a heterologous strain.

Apparently complete susceptibility to heterologous strains has been reported ${ }^{66,57}$, but this has also occurred with secondary homologous infections ${ }^{58}$. In 1936, Boyd and Kitchen ${ }^{56}$ reported that homologous immunity to $P$. falciparum was of little protective value against heterologous strains, but in 1945 they reversed this opinion ${ }^{54}$. The rate of onset of naturally acquired immunity against heterologous strains of $P$. falciparum may be similar to the rate of onset against homologous strains in syphilitic adults.

The large series of syphilis patients treated with a homologous strain of $P$. falciparum by Ciuca et al. ${ }^{15}$ (Fig. 1) was not repeated using heterologous isolates. The findings illustrated in Figs 5 and 6 imply that a similar rate of onset of protective immunity may have occurred had heterologous isolates been applied. The adult transmigrants from Java living in hyperendemic Irian Jaya model such a series. The incidence density of $P$. falciparum among adult male transmigrants from Java in the Arso region of northeastern Irian Jaya ranged from about two to three infections per person year 


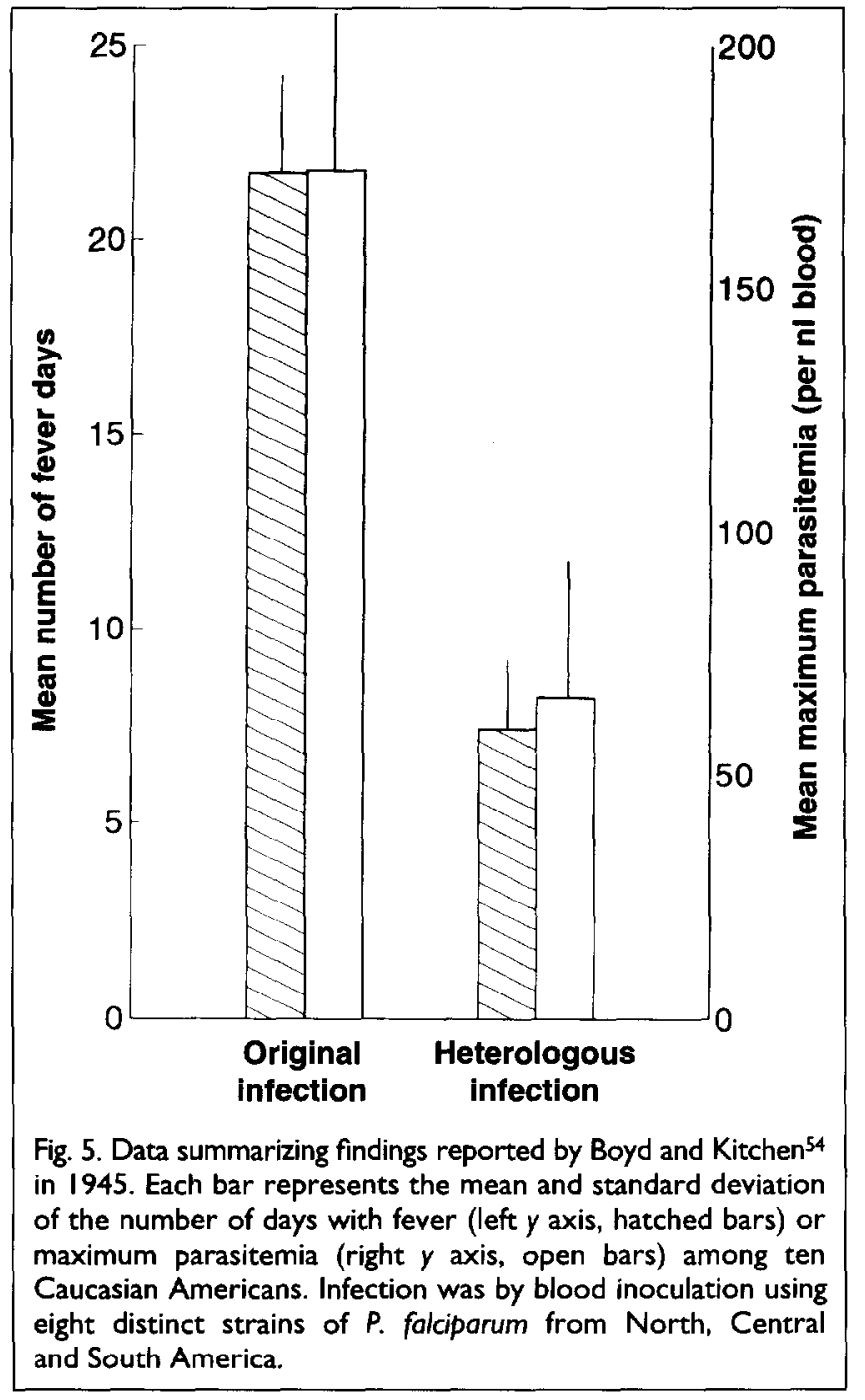

in one study ${ }^{59}$. These attack rates agreed with other estimates from the Arso region (J.K. Baird, PhD Thesis, Tulane University, 1994). If the onset of naturally acquired immunity in this region requires 12 to 24 months 8,10 , then roughly three to six infections occurred during this period. The rate of onset of protective immunity in these populations resembled that among adult syphilis patients exposed to serial inoculation by a single strain of $P$. falciparum.

\section{Geographically distinct strains of \\ Plasmodium falciparum}

If an age-dependent strain-transcending immune response is the basis of naturally acquired immunity, then adult immune sera should consistently inhibit the development of parasites from widely separated geographic regions. Precipitous decreases in densities of parasitemia by $P$. falciparum followed infusion of immune adult IgG from West Africa in children living in East Africa 60,61 . Several thousand kilometers between donor and recipient apparently had little effect upon the protective efficacy of immune adult IgG. This was also true when adult African IgG was injected into Aotus monkeys infected by Asian isolates of $P$. falciparum ${ }^{62}$. Druihle and colleagues injected IgG from immune adult Africans into acutely ill Thai patients with similarly dramatic effects $12,63,64$. Morcover, recrudescent parasitemias were equally responsive to a second course of therapy with the same IgG. The pronounced inhibi-

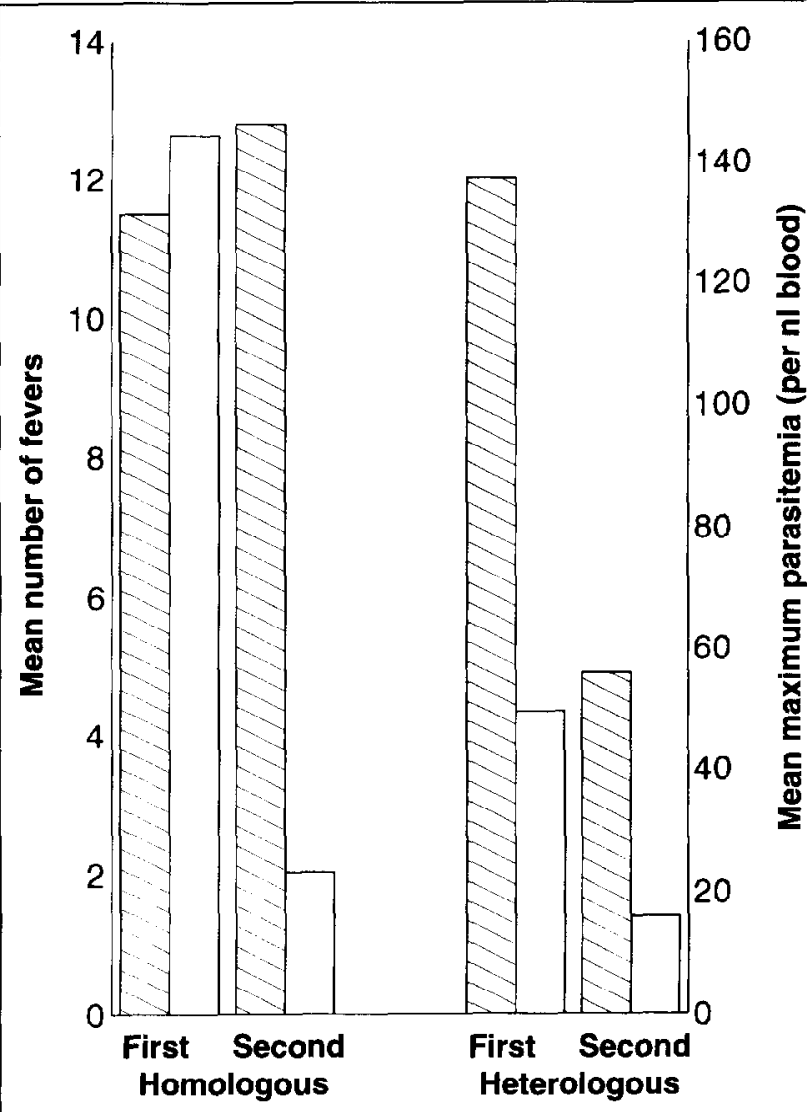

Fig. 6. Data summarizing findings reported by jeffery ${ }^{53}$ in 1966 The bars for the homologous $P$. falciparum infections represent the mean number of fevers (left $y$ axis, hatched bars) or mean maximum parasitemia (right $y$ axis, open bars) among four patients upon initial and secondary challenge. The bars for the heterologous infections represent the same data from seven patients. The five strains of $P$. falciparum used in these cxper iments came from North and South America and from Asia.

tory activity of African IgG against Thai isolates was confirmed in vitro (antibody-dependent cellular inhibition assay) ${ }^{65}$. Also, in vitro agglutination using sera and parasites from distant locations routinely occurred, including sera from Colombian adults with '... histories of exposure [to malaria] compatible with low-level immunity ${ }^{\prime 52}$. All of these observations point to a broad strain-transcending naturally acquired immunity among adults.

\section{Host age as a determinant of naturally acquired immunity}

The hypothesis of age-dependent naturally acquired immunity attributes protection in adults to only recent heavy exposure. In contrast, heavy exposure to infection among children, whether recent or lifelong, does not induce adult-like protection. Although antigenic polymorphism of the parasite may allow the proliferative course of $P$. falciparum in children, the constitution of the immune system of a child may be the basis of successful evasion of host defenses. The adult immune system appears capable of effective protection after brief heavy exposure (eg. five infections within 12 months).

Debate about a rapid rate of onset of naturally acquired immunity focused upon possible mechanisms of 'sufficient' exposure to antigenic polymorphism, 9,66 If onset in adults is indeed rapid, then the absence of 
adult-like protective immunity in children begs an explanation. After a brief period of apparently uniform heavy exposure among age groups, why are adults resistant to infection, while children remain susceptible? Constitutional differences between the immune systems of children and adults seems the most likely explanation. However, little is known of these differences, especially as regards immunity against infectious agents. Recognizing the basis of these differences may be another step toward understanding the cellular and molecular processes which govern naturally acquired immunity to $P$. falciparum.

\section{Acknowledgements}

The author gratefully acknowledges the Arso Malaria Team of the US Naval Medical Research Unit \#2, Jakarta, in particular Pak Purnomo and Hasan Basri. This team arid the staff which support thern are credited with the collection of data from Irian Jaya. The active interest, support and advice of Slamet Harjosuwamo and Budi Subianto of the Provincial Health Service in Irian Jaya has been essential to this laboratory since 1987. Discussions and correspondence with Mark A. James at Tulane University helped form the substance of this article. This work was supported by the Basic Studies and Threat Assessment work units of the US Naval Medical Research and Development Command, Bethesda, MD, USA. The views of the author are his own and do not purport to represent either those of the US Navy or those of the Department of Defense.

\section{References}

1 Bruce-Chwatt, L.J. (1980) Essential Malariology, Heinemann

2 McGregor, I.A. et al. (1956) Br. Med. J. 2, 686-692

3 McGregor, I.A. (1986) Clinics Trop. Med. Commun. Dis. 1, 29-53

4 Day, K.P. and Marsh, K. (1991) in Immunoparasitology loday (Ash, C. and Gallagher, R.B., eds), pp A68-A71, Elsevier Trends Journals, Cambridge

5 Hommel, M. (1985) Immunol. Today 6, 28-32

6 McGregor, I.A. and Wilson, R.J.M. (1988) in Malaria: Principles and Practice of Malariology (Wernsdorfer, W.H. and McGregor, I.A., eds), pp 559-619, Churchill Livingstone

7 Mendis, K.N. et al. (1991) in Immunoparasitology Today (Ash, C. and Gallagher, R.B., eds), pp A34-A37, Elsevier Trends Journals, Cambridge

8 Baird, J.K. et al. (1991) Am. J. Trop. Med. Hyg. 45, 65-76

9 Roberts, D.J. et al. (1993) Parasitology Today 9, 281-286

10 Baird, J.K. et al. (1993) Am. J. Trop. Med. Hyg. 49, 707-719

11 Roberts, D.J. et al. (1994) Parasitology Today 10, 64-65

12 Druihle, P. and Bouharoun-Tayoun, H. (1991) Res. Immunol. 142, $637-643$

13 Koch, R. (1900) Dtsche Med. Wochenschr. 26, 88-90

14 Boyd, M.F. (1949) Malariology (Vol. 1), WB Saunders

15 Ciuca, M. et al. (1934) Trans. R. Soc. Trop. Med. Hyg. 27, $619-622$
16 Yorke, W. and Macfie, J.W.S. (1924) Trans. R. Soc. Trop. Med. Hyg. 18, 13-44

17 Sinton, J.A. (1939) J. Mal. Insl. Ind. 2, 71-83

18 Brown, K.N. and Brown, I.N. (1965) Nature 208, 1286-1288

19 Handunnetti, S.M. et al. (1987) J. Exp. Med. 165, 1269-1283

20 Hommel, M. et al. (1983) J. Exp. Med. 157, 1137-1148

21 Biggs, B.A. et al. (1991) Proc. Natl Acad. Sci. USA 88, 9171-9174

22 Roberts, D.J. et al. (1992) Nature 357, 689-692

23 McBride, J.S. et al. (1982) Science 217, 254-257

24 McBride, J.S. et al. (1984) Trans. R. Soc. Trop. Med. Hyg. 78, 32-34

25 McBride, J.S. et al. (1985) J. Exp. Med. 161, 160-180

26 Creasey, A. et al. (1990) Am. J. Trop. Med. Hyg. 42, 403-413

27 Anders, R.F. (1986) Parasite Immunol. 8, 529-539

28 Schofield, L. (1991) Parasitology Today 7, 99-105

29 Wilson, B.D. (1936) Trans. R. Soc. Trop. Med. Hyg. 29, 583-617

30 Marsh, K. et al. (1989) Trans R. Soc. Trop. Med. Hyg. 83, 293-303

31 Chizzolini, C. et al. (1988) Am. J. Trop. Med. Hyg. 39, 150-156

32 Deloron, P. et al. (1987) Bull. WHO 65, 339-344

33 Petersen, E. et al. (1989) Am. J. Trop. Med. Hyg. 41, 386-394

34 Riley, E.M. et al. (1992) Parasite Immunol. 14, 321-337

35 McGregor, I.A. and Smith, D.A. (1952) Trans. R. Soc. Trop. Med. Hyg. 46, 403-427

36 Schuffner, W.A.P. (1939) J. Mal. Inst. India 1, 221-256

37 Christophers, S.R. (1924) Ind. J. Med. Res. 12, 273-294

38 Atmosoedjono, S. (1990) Wageningen Agricultural University Papers $90,141-167$

39 Zuckerman, A. and Yoeli, M. (1953) J. Infect. Dis. 94, 225-236

40 Singer, I. et al. (1955) J. Infect. Dis. 97, 15-21

41 Kasper, L.H. and Alger, N.E. (1973) J. Protozool. 20, 445-449

42 Cabrera, E.J. et al. (1973) J. Protozool. 20, 449-452

43 Spira, D.T. et al. (1970) Immunology 19, 759-766

44 Makinodan, T. et al. (1987) in Aging and the Immune Response Cellular and Humoral Aspects (Goidl, E.A., ed.), pp 27-43, Dekker

45 Thoman, M.L. and Weigle, W.O. (1989) Adv. Immunol. 46, 221-261 46 Hannet, I. et al. (1992) Immunol. Today 13, 215-218

47 Marsh, K. and Howard, R.J. (1986) Science 231, 150-153

48 Forsyth, K.P. et al. (1989) Am. J. Trop. Med. Hyg. 41, 259-265

49 Reeder, J.C. et al. (1994) Am. J. Trop. Med. Hyg. 51, 45-55

50 Gupta, S. et al. (1994) Science 263, 961-963

51 Newbold, C.I. et al. (1992) Exp. Parasitol. 75, 281-292

52 Aguiar, J.C. et al. (1992) Am. J. Trop. Med. Hyg. 47, 621-632

53 Jeffery, G.M. (1966) Bull. WHO 35, 873-882

54 Boyd, M.F. and Kitchen, S.F. (1945) J. Nat. Mal. Soc. 4, 301-306

55 Collins, W.E. et al. (1964) Am. J. Trop. Med. Hyg. 13, 777-782

56 Boyd, M.F. et al. (1936) Am. J. Trop. Med. 16, 139-145

57 James, S.P. et al. (1932) Proc. R. Soc. Med. 25, 1153-1158

58 James, S.P. and Shute, P.G. (1926) Report on the First Results of Laboratory Work on Malaria in England, pp 1-29, League of Nations Health Organization

59 Jones, T.R. et al. (1994) Am. J. Trop. Med. Hyg. 50, 210-218

60 Cohen, S. et al. (1961) Nature 192, 733-737

61 McGregor, I.A. (1964) Am. J. Trop. Med. Hyg. 13, 237-239

62 Diggs, C.L. et al. (1972) Proc. Helminthol. Soc. Wash. 39, 449-456

63 Sabchareon, A. et al. (1991) Am. J. Trop. Med. Hyg. 45, 297-308

64 Bouharoun-Tayoun, H. et al. (1990) J. Exp. Med. 172, 16331641

65 Lunel, F. and Druilhe, P. (1989) Infect. Immun. 57, 2043-2049

66 Gupta, S. and Day, K. (1994) Parasitology Today 10, 64 